Methods We comprehensively evaluated 68Ga-DOTATATE, a SST2 PET ligand, for imaging atherosclerosis. Target SSTR2 gene expression in macrophages and other immune cells were tested using population-based RNA-sequencing data. Patients with atherosclerosis $(n=42)$ underwent 68Ga-DOTATATE PET imaging in a prospective head-to-head comparison with $18 \mathrm{~F}$ FDG. 68Ga-DOTATATE autoradiography, immunostaining and quantitative PCR were performed in macrophages and excised carotid specimens from patients who underwent PET imaging. Results Target SSTR2 expression occurred exclusively in "proinflammatory" M1 macrophages, and no other cell type studied in vitro. In clinical imaging, 68Ga-DOTATATE mean of maximum tissue-to-blood ratios (mTBRmax) correctly identified culprit vs. non-culprit arteries in patients with acute coronary syndrome (median difference 0.69 [IQR 0.22 to 1.15 ], $\mathrm{p}=0.008$ ) and transient ischaemic attack or stroke (median difference 0.13 [IQR 0.07 to 0.32 ], $\mathrm{p}=0.003$ ). 68Ga-DOTATATE mTBRmax accurately predicted stable non-culprit coronary lesions with high-risk CT features (ROC AUC 0.86 [95\% CI 0.80 to 0.92], $\mathrm{p}<0.0001$ ), and correlated with Framingham risk score $(\mathrm{r}=0.53$ [95\% $\quad$ CI 0.32 to 0.69 ], $\mathrm{p}<0.0001)$ and vascular inflammation defined by $18 \mathrm{~F}-\mathrm{FDG}$ ( $\mathrm{r}=0.73$ [95\% CI 0.64 to 0.81 ], $\mathrm{p}<0.0001)$. While 18F-FDG mTBRmax also differentiated culprit from non-culprit carotid lesions (median difference 0.12 [IQR 0.0 to 0.23 ], $\mathrm{p}=0.008$ ) and high-risk from lower-risk coronary lesions (ROC AUC 0.76 [ $95 \%$ CI 0.62 to 0.91 ], $\mathrm{p}=0.002$ ), myocardial $18 \mathrm{~F}-\mathrm{FDG}$ spillover rendered coronary $18 \mathrm{~F}-\mathrm{FDG}$ scans uninterpretable in 27 (64\%) patients. In contrast, low myocardial 68Ga-DOTATATE binding allowed unimpeded coronary signal interpretation in all patients without the need for pre-scan fasting. Moreover, histological analysis confirmed specific binding of 68Ga-DOTATATE to SST2 receptors expressed by CD68-positive macrophages in excised carotid plaques. Carotid SSTR2 mRNA was highly correlated with both CD68 mRNA ( $\mathrm{r}=0.93$ [95\% CI 0.49 to 0.99 ]; $\mathrm{p}=0.007$ ) and in vivo 68Ga-DOTATATE PET signals measured from clinical images $(r=0.89$ [95\% CI 0.28 to 0.99], $\mathrm{p}=0.02$ ).

Conclusion We provide gene, cell, plaque and patient-level data, demonstrating that SST2 PET imaging using $68 \mathrm{Ga}-$ DOTATATE represents a macrophage-specific marker of atherosclerotic inflammation that outperforms 18F-FDG in the coronary arteries. Future research will explore the utility of 68Ga-DOTATATE inflammation imaging to classify high-risk patients for aggressive therapeutic intervention.

\section{E EOSINOPHILS HAVE AN ESSENTIAL ROLE IN CARDIAC REPAIR FOLLOWING MYOCARDIAL INFARCTION}

${ }^{1}$ Iqbal S Toor*, ${ }^{2}$ Dominik Rückerl, ${ }^{1}$ Adrian Thomson, ${ }^{3}$ Kare Tang, ${ }^{1}$ David E Newby, ${ }^{4}$ Adriano G Rossi, ${ }^{2}$ Judith E Allen, 'Gillian A Grey. 'BHF/University Centre for Cardiovascular Science, Queen's Medical Research Institute, University of Edinburgh, Edinburgh, UK; ${ }^{2}$ Faculty of Biology, Medicine and Health, School of Biological Sciences, University of Manchester, Manchester, UK; ${ }^{3}$ Essex Cardiothoracic Centre, Basildon and Thurrock Hospitals NHS Foundation Trust, London, UK; ${ }^{4}$ MRC Centre for Inflammation Research, Queen's Medical Research Institute, University of Edinburgh, Edinburgh, UK

\subsection{6/heartjnl-2017-311726.236}

Background Low peripheral blood eosinophil count is associated with increased risk of mortality in ischaemic heart disease patients. Eosinophils contain preformed IL-4 within their cytoplasmic granules, which promotes an anti-inflammatory response and is associated with tissue repair. Whether eosinophils are recruited to the infarct zone and have a role in regulating infarct repair is currently unknown.

Purpose This study sought to investigate the role of eosinophils in infarct healing.

Methods MI was induced by permanent coronary artery ligation in 12-15 week-old male wild-type (WT) BALB/c and eosinophil deficient $\Delta$ dblGATA mice. Cardiac function was assessed 7 days later by high-resolution ultrasound. A cohort of 732 patients undergoing emergency percutaneous coronary intervention for ST-elevation myocardial infarction (STEMI) were followed up for 6 month all-cause mortality.

Results Histochemical staining (Siglec $\mathrm{F}+$ ) and single cell digestion of infarcted WT $\mathrm{BALB} / \mathrm{c}$ hearts revealed significant recruitment of $(\mathrm{CD} 11 \mathrm{~b}+\operatorname{SiglecF}+\mathrm{Ly} 6 \mathrm{Gint})$ eosinophils to the infarcted heart. Genetic eosinophil deficiency in $\triangle$ dblGATA mice led to greater left ventricular dilatation relative to WT $\mathrm{BALB} / \mathrm{c}$ mice and worse cardiac function at Day 7 post-MI. Patients with a low eosinophil count at Day 1 following STEMI had an increased risk of 6 month all-cause mortality. On multivariate analysis the hazard ratio of all-cause mortality in the first tertile of peripheral blood eosinophil count at Day 1 post-MI was 6.97 [2.18-22.32] compared to the highest tertile $(p=0.001)$. Treatment with IL-4 complexes was able to rescue the adverse cardiac remodelling of eosinophil-deficient $\Delta$ dblGATA mice. Expression of plod2, lox and Fmod genes, which are involved in post-translational collagen modification, were increased in hearts from $\triangle$ dblGATA mice. This was accompanied with longer infarct length in $\triangle$ dblGATA mice compared to WT BALB/c mice $(\mathrm{p}=0.01)$.

Conclusions This study provides the first evidence for an essential role of eosinophils in the tissue repair response following MI, through regulating genes associated with connective tissue biogenesis. Patients with a low eosinophil post-MI may benefit from IL-4 therapy to improve outcome. 